\title{
Assessment the relationship between osteoporosis with tooth loss and dry mouth in women with osteoporosis
}

\begin{abstract}
Introduction: Osteoporosis is the most common metabolic bone disease and protests include decreased bone mineral and underlying tissue. Due to the prevalence of osteoporosis, especially in women and its complications, a study to evaluate the association of osteoporosis with tooth loss and oral dryness feeling in women with osteoporosis was designed.
\end{abstract}

Materials and methods: This cross sectional study was performed on 170 women referred to the center of bone densitometry of Fasa city. Data were collected using a valid questionnaire including standard questionnaire of xerostomia and demographic variables, also a clinical examination was carried out for determine the number of missing teeth. Then, the data were entered into the SPSS 19 and were analyzed. $\mathrm{P}<0.05$ was considered as statistically significant.

Results: The findings of this study showed that there was significant relationship between osteoporosis and oral dryness feeling $(\mathrm{p}<0.001)$. But no significant difference was found between the two groups regarding average number of teeth missing $(\mathrm{p}=0 / 09)$. However, significant difference was found between age and number of missing teeth $(\mathrm{p}=0 / 02)$.

Conclusion: The results showed that osteoporosis cannot be considered risk factors for tooth loss. But there is a positive relationship between osteoporosis and oral dryness feeling in women.

Keywords: osteoporosis, oral dryness, tooth loss, menopause, osteoporotic fractures, lactating
Volume 4 Issue 7 - 2016

\author{
Khiyali Z,' Kashfi SM,' Khani Jeihooni A,' Jari \\ Murjan $\mathrm{Y}^{2}$ Kashfi $\mathrm{SH}^{3}$ \\ 'Department of Public Health, School of Health, Fasa University \\ of Medical Sciences, Iran \\ ${ }^{2}$ Department of Medicine, Fasa University of Medical Sciences, \\ Iran \\ ${ }^{3}$ Faculty Member of Larestan Nursing School, Shiraz University \\ of Medical Sciences, Iran
}

Correspondence: Khani Jeihooni A, Department of Public health, School of Health, Shiraz University of Medical Sciences, Iran,Tel 09175328065,Email khiyaliz@yahoo.com

Received: June 06, 2016 | Published: November 22, 2016

\section{Introduction}

Osteoporosis is a serious problem in health care centers in developed and developing countries ${ }^{1}$ and since morbidity and high mortality due to this disease influence patient and society, it's considered as a global public health problem. ${ }^{2}$ According to studies, ten million people worldwide have osteoporosis and thirty-six million people suffer bone loss and by 2020 , almost 61 million people worldwide will have osteoporosis or loss of bone mass. ${ }^{3}$ Women, four times more than men are affected by osteoporosis, ${ }^{4}$ actually it is estimated that 200 million women worldwide suffer from osteoporosis. ${ }^{5}$ Statistics indicate that 1 in 3 women over age 50, as will 1 in 5 men aged over 50 have experienced osteopenia and osteoporotic fractures in Iran. ${ }^{6}$

Osteoporosis is a Multifactorial disease and low physical activity, poor nutrition, lack of calcium and vitamin $\mathrm{D}$, excessive alcohol consumption, smoking and other factors cause osteoporosis.? Although, this disease affects both males and females, osteoporosis in women is more than men (overall, $61 \%$ of osteoporotic fractures occur in women, with a female-to-male ratio of 1.6) because, bone structure changes very much with estrogen deficiency in postmenopausal that causes loss of bone mass and osteoporosis (estrogen deficiency following menopause or surgical removal of the ovaries is correlated with a rapid reduction in bone mineral density). On the other hand, hormonal changes during this period causes women become more susceptible to inflammatory diseases of the supporting tissues of the teeth; especially, if she doesn't regard the oral health, the risk of this disease will increase. ${ }^{8}$ Sultan ${ }^{9}$ showed in his studies that relation between bone density loss and bone cavity erosion, indicates that loss of bone mass during menopause have been as a serious factor for periodontal disease.

Periodontitis is a serious gum infection that damages the soft tissue and destroys the bone that supports your teeth. Periodontitis can cause tooth loss or worse, an increased risk of heart attack or stroke and other serious health problems. Periodontitis is usually the result of poor oral hygiene. Periodontitis involves progressive loss of the alveolar bone around the teeth, and if left untreated, can lead to the loosening and subsequent loss of teeth. Periodontitis is caused by microorganisms that adhere to and grow on the tooth's surfaces, along with an overaggressive immune response against these microorganisms. ${ }^{7}$ Many women with dry mouth go to the dental clinic to treat their problem ${ }^{10}$ and Hosseini's ${ }^{11}$ study showed that there is a relation between dry mouth and bone density and individuals with dry mouth suffer from osteoporosis, ${ }^{11}$ so this present study have been designed to relate between osteoporosis and tooth loss and dry mouth in women.

\section{Materials and methods}

This cross sectional study was performed on 170 women $40-60$ aged (85 with osteoporosis and 85 without osteoporosis) referred to the center of bone densitometry of Fasa city. Non-probability sampling method based on inclusion criteria (age range between 40 
and 60 years old, female sex, lack of blood diseases and systemic diseases such as diabetes, Sjogren's syndrome, etc. without the use of immune suppressive drugs such as corticosteroids, non-smoking, no history of periodontal surgery, lack of oral candidiasis, oral health optimal condition) was done among patients referred to the center of bone densitometry of Fasa city.

The bone density in the Hologic bones was determined based on DEXA using a L1-L4 densitometer. According to the World Health Organization, T-score $\geq 1$ as normal bone density, 1 T-score 2.5 as osteoporosis and T-score $<2.5$ as osteopenia ${ }^{12}$ and data of densitometry (BMD) included bone density in the lumbar spine and femoral area (thigh), healthy women and women with osteoporosis were identified. In this study, a standardized questionnaire for dry mouth was used and the individuals in the study were asked to complete it. Who respond to at least three questions positively, he is considered as patients with dry mouth ${ }^{11}$ and, if they don't respond to any question or three questions, they won't have dry mouth (xerostomia). Clinical examination was also carried out to determine the number of missing teeth. The collected data were analyzed by software SPSS19.

\section{Results}

Our findings showed that the mean and standard deviation of patients age in the group with osteoporosis with and without, respectively, was $35 / 6 \pm 05 / 52$ and $73 / 7 \pm 18 / 52$ that any significant difference was not observed between the two groups $(\mathrm{P}=0 / 44)$. Body mass index of the participants in the group with osteoporosis was $36 / 3 \pm 19 / 27$ and in the group without osteoporosis was $27 / 3 \pm 57 / 27$. Most of the participants had primary education (90/45 percent and $70 / 57$ percent in the group with osteoporosis and in the group without osteoporosis), respectively. In this study, significant differences were not observed between two groups in the parameters of family history in osteoporosis, history of fracture, lactating, menopause and underlying disease (thyroid disease or diabetes) (Table 1).

According to the studies in women with osteoporosis, there is not strong significant relationship between parameters such as age $(\mathrm{P}=0.55)$, body mass index $(\mathrm{P}=0.49)$ and education $(\mathrm{P}=0.36)$ with dry mouth. Table 2 showed comparison of the two groups in terms of dry mouth. Statistical studies showed that there was a significant relationship between osteoporosis and dry mouth in two groups with and without osteoporosis and there was statistically significant difference in term of dry mouth between two groups $(\mathrm{P}<0.001)$.

According to the studies in women with osteoporosis, it was observed a significant relationship between the number of missing teeth and the age $(\mathrm{P}=0.02)$ but it was not observed statistically significant relationship between the number of missing teeth and body mass index $(\mathrm{P}=0.73)$ and education $(\mathrm{P}=0.19)$. Table 3 showed three comparisons between two groups in terms of the number of missing teeth. Statistical analysis didn't show significant relationship between osteoporosis and the number of missing teeth in two groups with osteoporosis and without osteoporosis $(\mathrm{P}=0.09)$.

Table 1 Comparison of parameters between two groups in family history in osteoporosis, history of fracture, lactating, menopause and chronic diseases

\begin{tabular}{llllll}
\hline \multirow{2}{*}{ Group / Variable } & \multicolumn{2}{c}{ Without osteoporosis(n=85) } & \multicolumn{2}{c}{ With osteoporosis(n=85) } & \multirow{2}{*}{ Chi-square test } \\
\cline { 2 - 5 } & Percent & Frequency & Percent & Frequency & \\
\hline family history in osteoporosis & 16.5 & 14 & 12.9 & 11 & 0.19 \\
history of fracture & 12.9 & 11 & 12.9 & 11 & 1 \\
lactating & 2.34 & 2 & 1.17 & 1 & 0.24 \\
menopause & 70.6 & 60 & 64.7 & 55 & 0.1 \\
thyroid disease or diabetes & 11.8 & 10 & 15.11 & 13 & 0.18 \\
\hline
\end{tabular}

Table 2 Frequency distribution based on oral dryness and osteoporosis in two groups

\begin{tabular}{llll}
\hline Variable/Group & \multicolumn{2}{c}{ Oral dryness } & P-value \\
\cline { 2 - 3 } & Yes & No & \\
\cline { 1 - 2 } Without osteoporosis & $8(42.90 \%)$ & $77(58.90 \%)$ & P $<0.001$ \\
With osteoporosis & $57(67.05 \%)$ & $28(32.95 \%)$ & \\
\hline
\end{tabular}

Table 3 The average number of missing teeth in two groups with osteoporosis and without osteoporosis

\begin{tabular}{llll}
\hline \multirow{2}{*}{ Variable/Group } & \multicolumn{2}{l}{ The number of missing teeth } & P-value \\
\cline { 2 - 3 } & Average & Standard deviation & \\
\cline { 1 - 3 } Without osteoporosis & 6 & 2.11 & 0.09 \\
With osteoporosis & 7 & 1.01 & \\
& & &
\end{tabular}




\section{Discussion}

Many older people complain of oral dryness that is strongly associated with menopause, but the exact mechanism is not clear. The measurement of salivary flow associated with age changes and sex differences has been discussed in several studies. ${ }^{10}$ This study was done to investigate the relationship between osteoporosis and missing teeth and dry mouth in women referred to the center of bone densitometry of Fasa city. The results showed that women with osteoporosis have dry mouth, more than women without the disease. These results are similar to results of Agha-Hosseini's ${ }^{11}$ and Arbabi-Kalati's studies. ${ }^{10}$ Anil et al., ${ }^{13}$ studied on relationship between osteoporosis and oral diseases in 2013. Calcium accounts as the main mineral component of the human skeletal system. Resorption of bone thus can result in diffusion of calcium in to blood and further in to the saliva. ${ }^{14}$

Rabiei et al., ${ }^{15}$ compared 56 menopausal women with osteoporosis with 56 menopausal women without osteoporosis and showed that calcium concentration in women's saliva with osteoporosis that may indicate bone loss in these women. Although he hasn't investigated the presence of dry mouth, but it may be concluded that the reason of dry mouth in patients with osteoporosis in this study is likely higher concentration of calcium that this case needs to be investigated further. In this study was also observed that there was not significant relationship statistically between osteoporosis and the number of missing teeth in people with osteoporosis. During the studies done to investigate relationship between osteoporosis and missing teeth, it was seen different results. In Nicopoulou-Karayianni, ${ }^{16}$ Bollen et al., ${ }^{17}$ studies there was not also significant relationship between osteoporosis and tooth loss and this finding was in agreement with this work.

In Atroshkovite's ${ }^{18}$ studies Low bone mineral density (osteoporosis and bone mass loss during menopause) changed dental health and led to fall them rapidly, but this finding doesn't have any relationship with clinical and radiographic demonstration of chronic periodontitis. Darcy et al., ${ }^{19} \&$ Passos et al., ${ }^{20}$ indicate the relationship between osteoporosis and tooth loss, which does not match with our studies. In Lundstram's ${ }^{21}$ study also examined the relationship between osteoporosis and the number of missing teeth and the results showed no significant difference between osteoporosis and the number of missing teeth and results also match with the study.

Another finding of this study is a positive significant relationship between the age and the number of missing teeth, so if the age of people increases, the number of missing teeth will increase. This result was also observed in Roohafza ${ }^{22}$ \& Tsakos studies. ${ }^{23}$ Imirzalioglu et al., ${ }^{24}$ also introduced the age of people as an important risk factor for tooth loss. pourjavad et al., ${ }^{8}$ showed that there is not statistically significant differences between three groups with osteoporosis, low bone density and healthy people in average missing teeth of 60 women referred to the center of bone densitometry of Mashhad city.

Among the major limitations of this study, we can point to use questionnaire to investigate oral dryness. Using of saliva volume with questionnaires to determine the oral dryness has higher accuracy and precision. Since, using saliva to detect osteoporosis has several advantages: it's late-invasive and painless, trained personnel to collect saliva is not necessary, it is suggested that in future studies, the relationship between saliva and substances with osteoporosis is examined.

\section{Acknowledgements}

This study is thesis format of medical education of Yassin Jari
Murjani (NO: 94073) and it's sponsored by University of Medical Sciences of Fasa city, so I gratitude and appreciate them and women that assisted the research team.

\section{Conflict of interest}

The author declares no conflict of interest.

\section{References}

1. Cohen AJ, Roe FJC. Review of risk factors for osteoporosis with particular reference to a possible aetiological role of dietary salt. Journal of Food and Chemical Toxicology. 2000;38(2-3):237-253.

2. Matin N, Tabatabaie O, Keshtkar A, et al. Development and validation of osteoporosis prescreening model for Iranian postmenopausal women. Journal of Diabetes \& Metabolic Disorders. 2015;14:12-20.

3. National Osteoporosis Foundation (NOF). Physicians guide to prevention and treatment of osteoporosis. Washington DC, USA; 2002.

4. kathleen M, Stamp S, Bajan M, et al. Nutrition and diet therapy Krause s food nutrition. Pub Nutrition World. 2006;12:25.

5. Shirazi KK, Wallace LM, Niknami S, et al. A home-based, transtheoretical change model designed strength training intervention to increase exercise to prevent osteoporosis in Iranian women aged 40-65 years: a randomized controlled trial. Health Educ Res. 2007;22(3):305-317.

6. Pajouhi M, Komeylian Z, Sedaghat M, et al. Efficacy of educational pamphlets for improvement of knowledge and practice in patients with Osteoporosis. Payesh Journal of The Iranian Institute For Health Sciences Research. 2004;3(1):67-74.

7. Carranza FA, Newman MG, Takei HH. Clinical periodontology. 9th ed. London: WB Saunders; 2002. p. 204-523.

8. Pourjavad M, Moeintaghavi A, Dadgar S, et al. Assessment the Relationship between Osteoporosis with tooth loss in Postmenopausal Women. IJOGI. 2012;15(10):1-7.

9. Sultan N, Rao J. Association between periodontal disease and bone mineral density in postmenopausal women:a cross-sectional study. Med Oral Pathol Oral Cir Bucal. 2011;16(3):e440-e447.

10. Arbabi-Kalati F, Mohammadi A, Ghaemi Hesaroeeh S. Assessment the Relationship between Osteoporosis and Oral Dryness in Postmenopausal Women. J Mash Dent Sch. 2015;39(2):117-122.

11. Agha-Hosseini F, Mitzaii Dizgah I, Moosavi NM. Relationship of lumbar spine bone mineral density and oral dryness feeling in menopause. Menopause. 2011;18(6):625-628.

12. Stamp TCB Osteoporosis. In: Scott JT, editor. Copcrnan's Textbook of the Rheumatic disease. London: Churchil- 1 Livingstone; 1986:10041008.

13. Anil S, Preethanath RSS, AlMoharib HP, et al. Impact of Osteoporosis and Its Treatment on Oral Health. Am J Med Sci. 2013;346(5):396-401.

14. Kavitha MS, Asano A, Taguchi A, et al. Diagnosis of osteoporosis from dental panoramic radiographs using the support vector machine method in a computer aided system. BMC Medical Imaging. 2012;12(1):1-11.

15. Rabiei M, Masooleh IS, Leyli EK, et al. Salivary calcium concentration as a screening tool for postmenopausal osteoporosis. Int $J$ Rheum Dis. 2013;16(2):198-202.

16. Nicopoulou-Karayianni K, Tzoutzoukos P, Mitsea A, et al. Tooth loss and osteoporosis: the OSTEODENT Study. J Clin Periodontol. 2009;36(3):190-197.

17. Bollen AM, Taguchi A, Hujoel PP, et al. Number of teeth and residual alveolar ridge height in subjects with a history of self-reported osteoporotic fractures. Osteoporosis international. 2004;15(12):970 974. 
18. Atrushkevitch VG, Mlkrtumyan AM. Dental status of women in the post-menopase period. Gerontologija. 2009;10(3):135-142.

19. Darcey J, Horner K, Walsh T, et al. Tooth loss and osteoporosis: to assess the association between osteoporosis status and tooth number. British dental journal. 2013;214(4):e10.

20. Passos JS, Vianna MI, Gomes-Filho IS, et al. Osteoporosis/osteopenia as an independent factor associated with periodontitis in postmenopausal women:a case-control study. Osteoporos Int. 2013;24(4):1275-1283.

21. Lundstram A, Jendle J, Stenstram B, et al. Periodontal conditions in 70-year-old women with osteoporosis. Swed Dent J. 2001;25(3):89-96.
22. Roohafza H, Afghari P, Keshteli AH, et al. The relationship between tooth loss and psychological factors. Community Dent Health. 2015;32(1):16-9.

23. Tsakos G, Watt RG, Rouxel PL, et al. Tooth loss associated with physical and cognitive decline in older adults. J Am Geriatr Soc. 2015;63(1):91-99.

24. Imirzalioglu P, Yuzugullu B, Gulsahi A. Correlation between residua ridge resorption and radiomorphometric indices. Gerodontology. 2012;29(2):e536-e542. 